\title{
Temporal-mode continuous-variable cluster states using linear optics
}

\author{
Nicolas C. Menicucci \\ Perimeter Institute for Theoretical Physics, Waterloo, Ontario N2L 2Y5, Canada
}

(Dated: July 20, 2010)

\begin{abstract}
I present an extensible experimental design for optical continuous-variable cluster states of arbitrary size using four offline (vacuum) squeezers and six beamsplitters. This method has all the advantages of a temporalmode encoding [Phys. Rev. Lett. 104, 250503], including finite requirements for coherence and stability even as the computation length increases indefinitely, with none of the difficulty of inline squeezing. The extensibility stems from a construction based on Gaussian projected entangled pair states (GPEPS). The potential for use of this design within a fully fault tolerant model is discussed.
\end{abstract}

PACS numbers: 03.67.Lx, 42.50.Ex

\section{INTRODUCTION}

Quantum computation (QC) is the controlled coherent manipulation of quantum information. Analogous to its classical counterpart, quantum information is usually encoded in the quantum state of locally addressable systems, and its manipulation is performed by inducing coherent unitary evolution of these systems via external laboratory equipment [1]. The oneway model of QC [2] replaces this need for coherent unitary control by a sequence of adaptive local measurements made on a highly entangled resource state called a cluster state [3]. This resource acts as a universal substrate with quantum information encoded virtually within it. With local projective measurements often being easier to implement than unitary evolution, the philosophy of the model is this: perfect the creation of cluster states, and the rest is (comparatively) easy.

In its continuous-variable (CV) incarnation, universal oneway QC [4] requires a resource state known as a continuous variable cluster state [5], which is a multimode squeezed Gaussian state. In an optical setting, homodyne detection and photon counting - plus classical feedforward of measurement results-suffice to implement universal QC using CVs [6]. Homodyne detection alone is sufficient to implement all multimode Gaussian operations [6], given a cluster state with a sufficiently connected graph ${ }^{1}$

There are currently four proposed methods for constructing universal CV cluster states optically [4, 9, 11]. These all have the advantage of deterministic preparation over their optical qubit counterparts [12- -14$]$, which rely on nondeterministic interactions and postselection to generate the desired state. Each has specific advantages.

The canonical method [4] involves single-mode squeezers and controlled- $Z\left(\mathrm{C}_{Z}\right)$ gates, which are an example of a quantum nondemolition (QND) interaction [15]. The $\mathrm{C}_{Z}$ gate can be implemented using beamsplitters and inline squeezing (i.e., squeezing of a state other than the vacuum) [16, 17],

\footnotetext{
${ }^{1}$ While usage varies in the literature, I am using the convention [4] 7, [-10] that a "cluster state" can have any associated graph. Some authors prefer to call this a "graph state," reserving "cluster state" for square-lattice graph states only. I would refer to such states as "cluster states with a square-lattice graph."
}

which is experimentally challenging but achievable using current technology [18]. All $\mathrm{C}_{Z}$ gates commute, and this gate is the natural $\mathrm{CV}$ generalization of its qubit counterpart (used to create the qubit cluster states [3]), thus making this design particularly amenable to theoretical analysis.

The linear-optics method [11] eliminates the need for inline squeezing by replacing the $\mathrm{C}_{Z}$ gates with a network of beamsplitters. The replacement is not one for one, however, and an entirely new network will generally be needed to make a cluster state with a different graph (even if they differ by just one node). The major advantage of this method is that only vacuum (i.e., offline) squeezing and linear optics are needed, thus making this the method of choice in experiments to date [19. 23].

The single-OPO method [8, 9] combines all squeezing and interferometry into a single optical parametric oscillator (OPO), encoding the entire cluster state within a single beam. Each mode is an individual frequency within an optical frequency comb. The advantage of this method is its scalability. While the initial implementation is more complex than the linear-optics method, once the technology is established [2427], it is in principle much easier to scale up to cluster states that are larger by several orders of magnitude [9].

The single-QND-gate method [10] reintroduces the experimentally challenging $\mathrm{C}_{Z}$ gate. But in this case, only one such gate is needed because the modes are encoded temporally, each traversing the same optical path (but at different times) and each passing multiple times through the same optical hardware implementing the $\mathrm{C}_{Z}$ gate. This method has the additional advantage that the cluster state is extended as needed-simultaneously with measurements implementing an algorithm on it - in a manner analogous to repeatedly laying down additional track in front of a moving train car (a "Wallace and Gromit" approach; see footnote in Ref. [10]). Such a method eliminates the need for long-time coherence of a large cluster state because only a small piece of the state exists at any given time.

The current proposal is based on key elements from the last three. The linear-optics method [11] is used to generate states produced by the single-OPO method [8, 9] using the temporalmode encoding of the single-QND-gate method [10]. The current method improves over the single-QND-gate method by eliminating the need for inline squeezing (in the $\mathrm{C}_{Z}$ gate). 
The key observation that makes this simplification possible is that the states produced in the single-OPO method are Gaussian projected entangled pair states (GPEPS) [28]. GPEPS states can be described as a collection of entangled pairs linked together by projecting adjacent ends down to a lower-dimensional joint subspace (schematically, $\longrightarrow$ becomes $\longrightarrow \longrightarrow$ after projection on the circled nodes).

The paper is organized as follows. In Section II. I introduce the graphical formalism used for the main derivations. Section[III]describes a temporal-mode GPEPS-based construction of a CV quantum wire, and Section IV follows with the same for a square-lattice $\mathrm{CV}$ cluster state. Section $\mathrm{V}$ concludes with some discussion, including comments on fault tolerance and error correction in the CV one-way QC model.

\section{GRAPHS FOR GAUSSIAN PURE STATES}

\section{A. Basic properties}

To describe the states used in this work, I will use the graphical formalism that covers all Gaussian pure states in a unique and unified fashion [29]. This formalism is summarized here and simplified for the current presentation. The usual graphical formalism for CV cluster states [6, 30, 31] uses graphs with real-valued weights and is limited to representing ideal CV cluster states, which are necessarily infinitely squeezed and thus unphysical. Using the complex-matrix representation of Gaussian states [32], the graphical calculus for Gaussian pure states [29] generalizes these ideal CV cluster-state graphs to complex-weighted graphs that can be used to uniquely represent any Gaussian pure state 2 including approximate CV cluster states. In addition, other types of CV graphs, such as the $\mathcal{H}$-graphs used in the single-OPO method [9, 33], are incorporated directly into the formalism. Local and two-local Gaussian unitary operations, as well as local quadrature measurements, can be visualized as graph transformations.

To every $N$-mode Gaussian pure state $\left|\psi_{\mathbf{Z}}\right\rangle$ (ignoring overall displacement and up to an overall phase) we can uniquely assign an undirected, complex-wieghted graph on $N$ nodes whose adjacency matrix $\mathbf{Z}$ is an $N \times N$ complex symmetric matrix with positive-definite imaginary part. That is,

$$
\mathbf{Z}=i \mathbf{U}+\mathbf{V}
$$

where $\mathbf{U}=\mathbf{U}^{\mathrm{T}}$ and $\mathbf{V}=\mathbf{V}^{\mathrm{T}}$ are real, and $\mathbf{U}>0$. All Gaussian pure states have a uniquely associated graph of this form, and all graphs satisfying these conditions are uniquely associated with a Gaussian pure state [29]. This matrix arises naturally (up to an overall factor of $i$ ) in the position-space wavefunction for the state:

$$
\psi_{\mathbf{Z}}(\mathbf{q})=\frac{(\operatorname{det} \mathbf{U})^{1 / 4}}{\pi^{N / 4}} \exp \left[-\frac{1}{2} \mathbf{q}^{\mathrm{T}}(\mathbf{U}-i \mathbf{V}) \mathbf{q}\right]
$$

\footnotetext{
2 The formalism only describes the noise properties of Gaussian pure states [29]. Thus, overall displacements are not represented. From now on, I will assume that this caveat is understood.
}

where $\mathbf{q}$ is a column vector of c-numbers. Henceforth, I will unambiguously refer to "the Gaussian graph Z."

All Gaussian unitary operations can be represented by the action of a symplectic matrix

$$
\mathbf{S}=\left(\begin{array}{ll}
\mathbf{A} & \mathbf{B} \\
\mathbf{C} & \mathbf{D}
\end{array}\right)
$$

on a column vector of Heisenberg-picture operators $\hat{\mathbf{x}}=$ $\left(\begin{array}{c}\hat{\mathbf{q}} \\ \hat{\mathbf{p}}\end{array}\right)$, where $\hat{\mathbf{q}}=\left(\hat{q}_{1}, \ldots, \hat{q}_{N}\right)^{\mathrm{T}}$, and $\hat{\mathbf{p}}=\left(\hat{p}_{1}, \ldots, \hat{p}_{N}\right)^{\mathrm{T}}$, with $\hat{\mathbf{x}}^{\prime}=\mathbf{S x}$. The new Gaussian graph $\mathbf{Z}^{\prime}$ associated to the resulting Gaussian pure state satisfies

$$
\mathbf{Z} \stackrel{\mathbf{S}}{\longrightarrow} \mathbf{Z}^{\prime}=(\mathbf{C}+\mathbf{D Z})(\mathbf{A}+\mathbf{B Z})^{-1},
$$

which can be interpreted as a (generally rather complicated) graph transformation rule. However, since all general Gaussian unitary operations can be decomposed into a sequence of local and 2-local Gaussian unitaries chosen from a fiducial set, we can build up complicated graph transformations by repeated application of simpler ones. Ref. [29] has more details on the general case; we will use a simplified version.

The Gaussian pure state $\left|\psi_{\mathbf{Z}}\right\rangle$ satisfies the following nullifier relation with respect to its graph $\mathbf{Z}$ :

$$
(\hat{\mathbf{p}}-\mathbf{Z} \hat{\mathbf{q}})\left|\psi_{\mathbf{Z}}\right\rangle=0,
$$

where the entries in $\hat{\mathbf{p}}$ and $\hat{\mathbf{q}}$ are to be interpreted as Schrödinger-picture operators in this context because the state $\left|\psi_{\mathbf{z}}\right\rangle$ is indicated explicitly ${ }^{3}$ The vacuum (i.e., ground state) on all $N$ modes is represented by $\mathbf{Z}=i \mathbf{I}$, a completely disconnected graph with only self-loops of weight $i$. It is clear that Eq. (5) holds for this state because $\hat{p}_{j}-i \hat{q}_{j}=-i \sqrt{2} \hat{a}_{j}$, where $\hat{a}_{j}$ is the annihilation operator for mode $j$. In the limit that $\mathbf{U} \rightarrow \mathbf{0}$, the Gaussian graph $\mathbf{Z}$ limits to a real-weighted ideal CV cluster-state graph $\mathbf{V}$, and Eq. (5) becomes the usual nullifier relation for these states [6]. For our purposes, we would also like to represent the $\mathcal{H}$-graph states of the singleOPO method [33]. These states have $\mathbf{V}=\mathbf{0}$. Given an $\mathcal{H}$ graph $\mathbf{G}$, the associated Gaussian graph is

$$
\mathbf{Z}=i e^{-2 \alpha \mathbf{G}},
$$

where $\alpha>0$ is an overall squeezing parameter.

$\mathcal{H}$-graphs are incorporated into this graphical formalism through the matrix exponential map, as in Eq. (6), which is normally a highly nontrivial operation. However, it simplifies under certain conditions. First of all, note that when $\mathbf{G}$ is $b i$ partite (i.e., its nodes can be separated completely into two groups such that no edge links nodes of the same group), it can be written as

$$
\mathbf{G}=\left(\begin{array}{cc}
\mathbf{0} & \mathbf{G}_{0}^{\mathrm{T}} \\
\mathbf{G}_{0} & \mathbf{0}
\end{array}\right)
$$

\footnotetext{
${ }^{3}$ Notice that this is a literal equality, unlike the relation of similar form, $\hat{\mathbf{p}}-\mathbf{A} \hat{\mathbf{q}} \rightarrow 0$, for ideal CV cluster states with graph $\mathbf{A}$, which is strictly true only in the limit of infinite squeezing [6].
} 
When $\mathbf{G}$ is additionally self-inverse (i.e., $\mathbf{G}^{2}=\mathbf{I}$ ), then $\mathbf{G}_{0}$ is square and satisfies $\mathbf{G}_{0}^{\mathrm{T}} \mathbf{G}_{0}=\mathbf{G}_{0} \mathbf{G}_{0}^{\mathrm{T}}=\mathbf{I}$. In this case, Eq. (6) simplifies to

$$
\begin{aligned}
\mathbf{Z} & =i \cosh 2 \alpha \mathbf{I}-i \sinh 2 \alpha \mathbf{G} \\
& =\left(\begin{array}{cc}
i \cosh 2 \alpha \mathbf{I} & -i \sinh 2 \alpha \mathbf{G}_{0}^{\mathrm{T}} \\
-i \sinh 2 \alpha \mathbf{G}_{0} & i \cosh 2 \alpha \mathbf{I}
\end{array}\right) .
\end{aligned}
$$

The essence of the $\mathcal{H}$-graph construction method is that this state is equivalent to an approximate CV cluster state after phase shifting one of the two sets of nodes (with respect to the graph bipartition) [9, 33]. Performing a Fourier transform (i.e., phase shift by $-\frac{\pi}{2}$ ) on the first half of the nodes gives [29]

$$
\mathbf{Z}^{\prime}=\left(\begin{array}{cc}
i \operatorname{sech} 2 \alpha \mathbf{I} & \tanh 2 \alpha \mathbf{G}_{0}^{\mathrm{T}} \\
\tanh 2 \alpha \mathbf{G}_{0} & i \operatorname{sech} 2 \alpha \mathbf{I}
\end{array}\right),
$$

which is indeed an approximate CV cluster state since $\mathbf{Z}^{\prime} \rightarrow$ $\mathbf{G}$ in the infinite-squezing limit $(\alpha \rightarrow \infty))^{4}$ The fact that the ideal CV cluster state approximated by $\mathbf{Z}^{\prime}$ has the same graph as the $\mathcal{H}$-graph $\mathbf{G}$ is a peculiar feature of bipartite, selfinverse $\mathcal{H}$-graphs [9, 34]; usually the graphs are very different [33].

\section{B. $\mathcal{H}$-graph states made without an $\mathcal{H}$-graph}

At this point, we should forget about the $\mathcal{H}$-graph method of construction for $\mathbf{Z}$. It doesn't matter how the state represented by Eq. (8) was created-all we care about is that the graph for it is given by $\mathbf{Z}$ as shown, and we will use graphs of this form even when $\mathbf{G}$ is only approximately self-inverse (due to imperfections at the boundary of a line or lattice). This is an important distinction because the simplification of the exponential map only holds when $\mathbf{G}$ is exactly self-inverse. Stray connections and imperfections in the $\mathcal{H}$-graph will generally "bleed out" via the exponential and contaminate connections all over the Gaussian graph $\mathbf{Z}$, which will end up failing to produce the state that we want, Eq. (8), and without an easy way to isolate and eliminate the bad links. If instead we start directly from Eq. [8], then we'll find-when we phase shift the nodes as above- that imperfections in $\mathbf{Z}$ are confined to a small neighborhood of the imperfections in $\mathbf{G}$. In fact, in this case, we have [29]

$$
\begin{aligned}
& \mathbf{Z}^{\prime}= \\
& \left(\begin{array}{cc}
i \operatorname{sech} 2 \alpha \mathbf{I} & \tanh 2 \alpha \mathbf{G}_{0}^{\mathrm{T}} \\
\tanh 2 \alpha \mathbf{G}_{0} & i \operatorname{sech} 2 \alpha\left[\cosh ^{2} 2 \alpha \mathbf{I}-\sinh ^{2} 2 \alpha \mathbf{G}_{0} \mathbf{G}_{0}^{\mathrm{T}}\right]
\end{array}\right),
\end{aligned}
$$

\footnotetext{
${ }^{4}$ Note that this is an approximate CV cluster state despite the fact that it is inequivalent to the analogous approximate $\mathrm{CV}$ cluster state made by the canonical method [4], whose Gaussian graph would be $\mathbf{Z}=i e^{-2 r} \mathbf{I}+\mathbf{G}$. In this expression, $r$ is the single-mode squeezing parameter for all the nodes that then pass through a collection of $\mathrm{C}_{Z}$ gates in accord with $\mathbf{G}$. One major advantage of the unified Gaussian graphical formalism is the ability to distinguish between these distinct approximants to the same ideal CV cluster state.
}

which reduces to Eq. 97 when $\mathbf{G}$ is self-inverse.

Consider the case in which only a small collection of nodes prevents $\mathbf{G}$ from being strictly self-inverse. Because the matrix $\mathbf{Z}^{\prime}$ in Eq. (10) involves the square of $\mathbf{G}$ (through the term $\mathbf{G}_{0} \mathbf{G}_{0}^{\mathrm{T}}$ ), nodes with incorrect edges in $\mathbf{G}$ can only affect other nodes that are at most two steps away (rather than everywhere, in the case of the exponential). To see this, define $\mathbf{G}=\overline{\mathbf{G}}+\mathbf{E}$, where $\overline{\mathbf{G}}$ is bipartite and exactly selfinverse, and $\mathbf{E}$ is bipartite (with the same partitioning), real, symmetric, and sparse. In addition, both $\overline{\mathbf{G}}$ and $\mathbf{E}$ have an analogous decomposition to that of $\mathbf{G}$ in Eq. (7), and therefore $\mathbf{G}_{0}=\overline{\mathbf{G}}_{0}+\mathbf{E}_{0}$, as well. $\mathbf{E}$ is a matrix of just a few "errors" that cause $\mathbf{G}$ to fail to be perfectly self-inverse. We have the following relation:

$$
\begin{aligned}
\mathbf{G}_{0} \mathbf{G}_{0}^{\mathrm{T}} & =\left(\overline{\mathbf{G}}_{0}+\mathbf{E}_{0}\right)\left(\overline{\mathbf{G}}_{0}+\mathbf{E}_{0}\right)^{\mathrm{T}} \\
& =\mathbf{I}+\mathbf{E}_{0} \overline{\mathbf{G}}_{0}^{\mathrm{T}}+\overline{\mathbf{G}}_{0} \mathbf{E}_{0}^{\mathrm{T}}+\mathbf{E}_{0} \mathbf{E}_{0}^{\mathrm{T}} .
\end{aligned}
$$

Now define $\mathbf{P}_{0}$ to be a matrix with the same dimensions as $\mathbf{E}_{0}$ that is constructed in the following way: first, place a 1 in the diagonal entry $\left(P_{0}\right)_{j j}$ if row $j$ of $\mathbf{E}_{0}$ consists of all zeros (i.e., if $\left(E_{0}\right)_{j k}=0 \forall k$ ), and place 0 's everywhere else; then, remove the all-zero rows of $\mathbf{P}_{0}$. Notice that $\mathbf{P}_{0} \mathbf{E}_{0}=\mathbf{0}$, which gives

$$
\mathbf{P}_{0} \mathbf{G}_{0} \mathbf{G}_{0}^{\mathrm{T}} \mathbf{P}_{0}^{\mathrm{T}}=\mathbf{P}_{0} \mathbf{P}_{0}^{\mathrm{T}}=\mathbf{I},
$$

where the identity matrix on the right is smaller than that in Eq. (11). Now further define

$$
\mathbf{P}:=\left(\begin{array}{cc}
\mathbf{I} & \mathbf{0} \\
\mathbf{0} & \mathbf{P}_{0}
\end{array}\right),
$$

which is also not square, where the identity matrix is the same size as $\mathbf{E}_{0}$, and the blocks of 0 are sized appropriately $5^{5}$

Measurements of $\hat{q}_{j}$ delete a node $j$ and all of its links from a given Gaussian graph [29], which is equivalent to deleting the $j^{\text {th }}$ row and column from its adjacency matrix. Conjugating $\mathbf{Z}^{\prime}$ from Eq. 10, by $\mathbf{P}$ deletes from $\mathbf{Z}^{\prime}$ the rows and columns that correspond to the nonzero rows of $\mathbf{E}_{0}$ (and, equivalently, the nonzero columns of $\mathbf{E}_{0}^{\mathrm{T}}$ ):

$$
\begin{aligned}
\mathbf{P Z}^{\prime} \mathbf{P}^{\mathrm{T}} & =\left(\begin{array}{cc}
i \operatorname{sech} 2 \alpha \mathbf{I} & \tanh 2 \alpha \mathbf{G}_{0}^{\mathrm{T}} \mathbf{P}_{0}^{\mathrm{T}} \\
\tanh 2 \alpha \mathbf{P}_{0} \mathbf{G}_{0} & i \operatorname{sech} 2 \alpha \mathbf{I}
\end{array}\right) \\
& =i \operatorname{sech} 2 \alpha \mathbf{I}+\tanh 2 \alpha \mathbf{P} \mathbf{G} \mathbf{P}^{\mathrm{T}},
\end{aligned}
$$

where Eq. (12) has been used. This is an approximate CV cluster state with most (but not all) of the graph $\mathbf{G}$ intact; the troublesome links have been deleted by deleting nodes that they attach to. In this case, all of the deleted nodes are taken

\footnotetext{
${ }^{5}$ Alternatively, $\mathbf{P}$ can be defined directly in terms of the Moore-Penrose pseudoinverse of $\mathbf{E}_{0}$, which is denoted $\mathbf{E}_{0}^{+}$. Starting with

$$
\left(\begin{array}{cc}
\mathbf{I} & \mathbf{0} \\
\mathbf{0} & \mathbf{I}-\mathbf{E}_{0} \mathbf{E}_{0}^{+}
\end{array}\right)
$$
}

which is square, we can form $\mathbf{P}$ simply by removing the all-zero rows. 
from the second set of the graph bipartition. When these nodes are located near the boundary of a regular lattice (or otherwise isolated in a graph with local topology), then deleting these nodes (or ones just to the "inside" of them) using $\hat{q}$ measurements is all that is needed to eliminate the imperfections.

The point of the preceding analysis is to justify ignoring the irregular boundary conditions in the sections that follow. In the single-OPO scheme, these boundary irregularities in $\mathbf{G}$ could not be ignored because they would contaminate the entire state through the exponential map. In this case, they can be dealt with simply by deleting nearby nodes. This is analogous to using scissors to clean up the jagged edge of a torn piece of paper.

\section{Simplified graphical formalism}

There are only three graph transformation rules that we will need for this work: (1) measurements of $\hat{q}$, (2) phase-plane inversion (i.e., phase shift by $\pi$ ), and (3) interference through a 50:50 beamsplitter. All of the graphs we use will be of the form of Eq. (8), but we will make the following modifications to the general formalism [29]. First, we will not draw the selfloops indicated by the diagonal of $\mathbf{Z}$, but we will be mindful of them when we derive the simplified graph rules that follow. Second, colors indicate the sign of the edge weight but not its actual value, with

$$
==\mp i \mathcal{C} \sinh 2 \alpha \quad \text { in the graph } \mathbf{Z},
$$

where $\mathcal{C}>0$. Nevertheless, for the graphs used in this work, the following relation often (but not always) holds:

$$
\mathcal{C}=\max \left(d_{1}, d_{2}\right)^{-\frac{1}{2}}
$$

where $d_{1,2}$ are, respectively, the degrees (number of neighbors) of the two nodes linked by the edge in question ${ }^{6}$ Within the approximate $\mathrm{CV}$ cluster state $\mathbf{Z}^{\prime}$ that eventually results after the phase shifts, these weights become

$$
=\mapsto \pm \mathcal{C} \tanh 2 \alpha \stackrel{\alpha \rightarrow \infty}{\longrightarrow} \pm \mathcal{C} \quad \text { in the graph } \mathbf{Z}^{\prime}
$$

with $\alpha \rightarrow \infty$ indicating the infinite-squeezing limit. The fact that only the sign of the weights is indicated visually does not take away from the rigor of these results; it is merely for visual simplicity. When relevant, the actual edge weights will be specified.

Finally, the bipartite nature of $\mathbf{Z}$ will be indicated by coloring the nodes black and white, according to the division into two sets. This coloring is only a visual distinction and has

\footnotetext{
${ }^{6}$ This seemingly strange formula arises because of the need for $\mathbf{G}$ in Eq. 8) to be self-inverse almost everywhere (see Section IIB). The exception to Eq. 16] is when $\hat{q}$ measurements are used to delete nodes and their edges from the graph; the remaining edges retain their weights before the deletion.
}

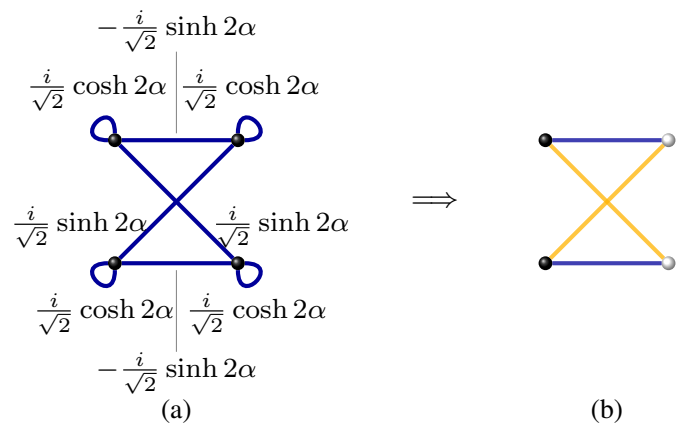

FIG. 1. Simplified graphical formalism for the Gaussian pure states represented in this paper. (a) Full graph for a particular Gaussian pure state [29]. (b) Simplified graph for the same state, as used in this paper. Self loops are not drawn; colored edges indicate the sign of the weights as in Eq. 15, with $\mathcal{C}=\frac{1}{\sqrt{2}}$ (see text) for all edges since each edge connects nodes having exactly 2 neighbors; and colored nodes indicate the bipartite splitting, with white nodes receiving a Fourier transform to convert this to an approximate CV cluster-state with the same graph, but now with weights given by Eq. 17.

no physical significance on its own $7^{7}$ The white nodes will eventually be Fourier transformed (i.e., phase shifted by $-\frac{\pi}{2}$ ) to implement the map $\mathbf{Z} \mapsto \mathbf{Z}^{\prime}$, as in Eq. (9), but since all of the graphs we will discuss satisfy $\mathbf{Z} \sim \mathbf{Z}^{\prime} \sim \mathbf{G}$ up to an affine transformation (and neglecting boundary irregularities), we will not need a graph transformation for the Fourier transform. In addition, since $\mathrm{CV}$ cluster states are used for one-way QC by making local measurements only, the Fourier transform can be absorbed into the eventual measurement procedure and becomes simply a change of basis ${ }^{8}$ An example of this simplification of the Gaussian graph formalism is presented in Figure 1 .

Below are the graph transformations used in this work. These rules are rigorous for the graphs considered in this work and are derived from the general transformation rules for Gaussian graphs [29], but they have been adapted and simplified for the representation used here. These rules function in exactly the same way on both black and white nodes.

Measurement of $\hat{q}$-As discussed above, this operation simply deletes the measured node and its links from the Gaussian graph $\mathbf{Z}$. The measured node is indicated by a small arrow:

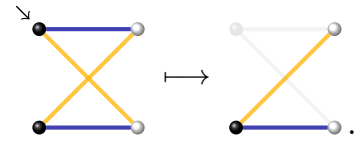

\footnotetext{
${ }^{7}$ In particular, while different colors were used in Ref. [29] to distinguish the meaning of nodes in ideal CV cluster-state graphs from their meaning in general Gaussian graphs, both colors of nodes in the present paper correspond to black nodes in Ref. [29]. All of the graphs used here are simplified versions of general Gaussian graphs.

${ }^{8}$ Therefore, when we talk about, for example, measuring a white node of the resulting CV cluster state in $\hat{q}$, we really mean measuring the same node of the original state in $\hat{F}^{\dagger} \hat{q} \hat{F}=-\hat{p}$.
} 
Recall that the colors only indicate the sign of the edge weights. The actual values of these weights do not change with the deletion, but it is worth emphasizing that they may no longer satisfy Eq. (16).

Phase-plane inversion (phase shift by $\pi$ ) —This operation is also very simple. All edges attached to the inverted node (indicated by highlighting) change color:

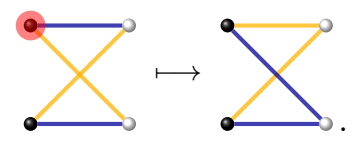

This corresponds to the edge weight changing sign but not magnitude.

50:50 beamsplitter-The 50:50 beamsplitter implements $\mathbf{S}_{\mathrm{BS}}\left(\frac{\pi}{4}\right)$ from Ref. [29]:

$$
\mathbf{S}_{\mathrm{BS}}\left(\frac{\pi}{4}\right)=\left(\begin{array}{cccc}
\frac{1}{\sqrt{2}} & -\frac{1}{\sqrt{2}} & 0 & 0 \\
\frac{1}{\sqrt{2}} & \frac{1}{\sqrt{2}} & 0 & 0 \\
0 & 0 & \frac{1}{\sqrt{2}} & -\frac{1}{\sqrt{2}} \\
0 & 0 & \frac{1}{\sqrt{2}} & \frac{1}{\sqrt{2}}
\end{array}\right) .
$$

Under the conditions of no link between the nodes being interfered and equal self-loop weights (which is the case with all graphs in this paper but is not displayed in the simplified formalism), we obtain the following very simple rules:
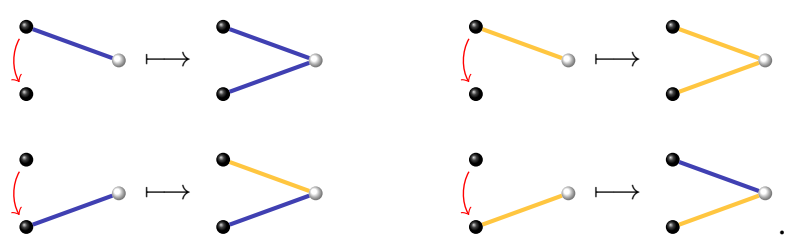

Notice that the beamsplitter duplicates a single link, but multiplies the magnitude of each by a factor of $\frac{1}{\sqrt{2}}$ in the process. The arrow points from node 1 to node 2 of the interaction, distinguishing the effect of the negative signs in Eq. (18). When an edge is copied in the direction of the arrow, the same color is applied to the new edge. When it is copied in the opposite direction, the new edge has the opposite color. Even with their simplified presentation, all of these graph transformation rules are rigorous for the graphs used in this paper.

\section{GPEPS QUANTUM WIRE}

The single-OPO "quantum wire"-a cluster state with a one-dimensional topology — actually has a width of two nodes and looks like this [9]:

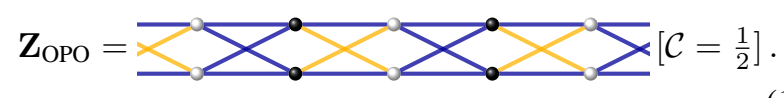

Notice that only a portion of the full graph is displayed 9 We can perform a $\pi$ phase shift on every other node on the top row

\footnotetext{
${ }^{9}$ If the full graph has periodic boundary conditions (or if it is formally infinite), then $\mathbf{G}$ for it is self-inverse [9], and Eq. 9] is satisfied. If, however,
}

to put this state into a translationally invariant form (ignoring node color, which has no physical significance):

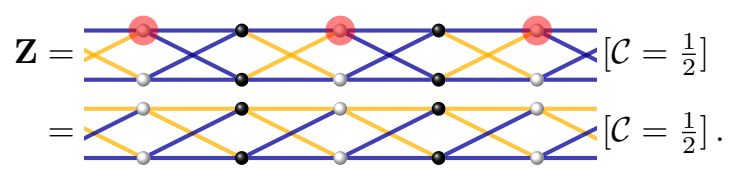

The connection to a GPEPS construction is immediate when we notice that this is exactly the result of applying the 50:50 beamsplitter transformation rules to a collection of two-mode squeezed states arranged as follows:

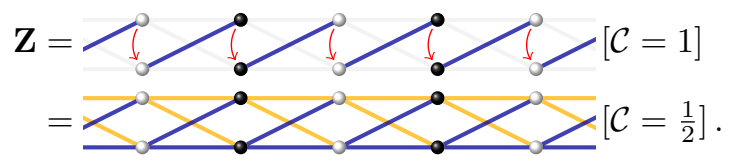

Notice that the order of application of these beamsplitters does not matter; the rules commute and result in the same graph. The Gaussian graph $\mathbf{Z}$ is the desired output of a temporalmode linear-optics construction method. Once in this form, measurements of $\hat{q}$ can be be made on all upper nodes (following the implicit phase shift by $-\frac{\pi}{2}$ on all white nodes; see Section IIC, projecting the bottom row of nodes into an ordinary (one-dimensional) approximate CV quantum wire [6]:

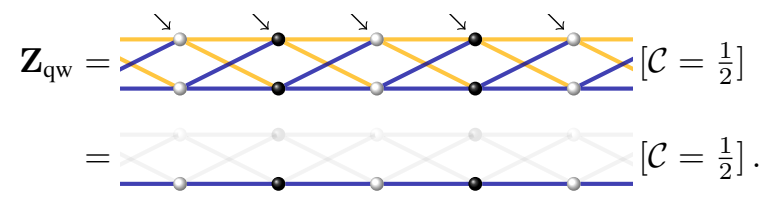

This GPEPS-based construction method has a simple and natural implementation using single-mode squeezers and linear optics. This method uses a temporal-mode encoding [10], which gives it all the advantages of the equivalent method using a single QND gate (in terms of coherence requirements and extensibility) but improves on it because all of the required squeezing is offline. The method is illustrated in Figure 2.

A few important notes are in order. First, periodic boundary conditions are used in the single-OPO construction [9] to strictly enforce the requirement that $\mathbf{G}^{2}=\mathbf{I}$, which otherwise fails at the ends. As shown in Section II B, however, in the construction presented here (which does not rely on exponentiating an $\mathcal{H}$-graph) these "contaminated" nodes can be disconnected from the rest of the cluster state by appropriate measurements of $\hat{q}$ on neighboring nodes, just like they are in the single-QND-gate method [10].

Second, it may not be necessary to do the projection of (the upper) half of the nodes in $\hat{q}$. This was used simply to prove that the cluster state with graph $\mathbf{G}$ can serve as a CV quantum wire. Instead, one might be able to use the entirety of $\mathbf{G}$ as the quantum wire, with quantum information encoded in an

the graph has at least one boundary, then only Eq. 10] is satisfied. As we have already seen in Section II B these boundary effects can be dealt with by suitably clipping the graph using $\hat{q}$ measurements. 


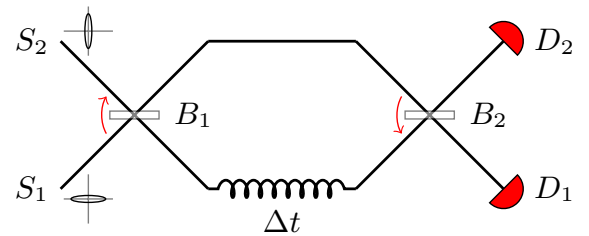

FIG. 2. Temporal-mode GPEPS construction of a CV quantum wire using passive squeezing and linear optics. Two single-mode squeezers $S_{1}$ and $S_{2}$ generate vacuum $\hat{p}$ - and $\hat{q}$-squeezed pulses of light (respectively, as shown) at regular intervals $\Delta t$. These pass through a simple 50:50 beamsplitter $B_{1}$, resulting in a two-mode squeezed state. (Red arrows point from the first node to the second in Eq. 18. for each beamsplitter.) The delay loop in the bottom line delays the bottom mode by $\Delta t$, allowing it to match up with the top mode of the subsequent pair emerging from $B_{1}$, resulting schematically in the graph shown in Eq. 21. The second 50:50 beamsplitter $B_{2}$ implements sequentially each of the transformations indicated by the red arrows, resulting in the final graph of Eq. 22). These pulses head toward detectors $D_{1}$ and $D_{2}$, which implement the necessary $\hat{q}-$ measurements (phase shifted as appropriate for the white nodes; see Section II C, which are indicated by the arrows in Eq. [23, as well as the adaptive measurement-based quantum algorithm to be implemented on the one-dimensional CV quantum wire. The adaptiveness means that subsequent measurement bases generally must be chosen based on previous measurement outcomes. Most measurements will involve homodyne detection in a basis that must be calculated and updated before the arrival of the next pulse, but the ability to divert the beam to an efficient photon counter is also required for universal single-mode QC [6].

appropriate joint subspace of each two-node pair. It is an open problem to determine the optimal measurements to perform in this case, but initial results indicate the possibility. There are several reasons to think this would be useful. First, the final cluster state in the construction above has edges with $\mathcal{C}=\frac{1}{2}$, while the two-mode squeezed states all had $\mathcal{C}=1$. While this has no effect on proofs of universality (since a non-unit edge weight simply corresponds to squeezing of the quantum information as it passes through the cluster), it could have important effects on the efficiency of the scheme with respect to the amount of initial entanglement in the two-mode squeezed states, and weights close to \pm 1 in the final CV cluster-state graph are preferred [33]. Also, with a joint subspace between vertical node pairs now carrying the quantum information, the other (unused) joint subspace might play an error correcting role. Error correction and fault tolerance for CV one-way QC remains an important open problem [28] and will be discussed further in Section $\nabla$.

\section{GPEPS SQUARE-LATTICE CLUSTER STATE}

The GPEPS techniques developed above for the CV quantum wire can be adapted to a two-dimensional square-lattice $\mathrm{CV}$ cluster state, as well. This additional dimension makes the state universal for CV one-way QC [4]. We again start with the states created by the single-OPO method [8, 9], which have the local topology of a square lattice, but with four phys- ical nodes per site of the lattice. Analogous to the quantum wire, we can make this graph invariant under translations in either lattice dimension by phase shifting the highlighted nodes by $\pi$ (which, again, is a local redefinition of basis only):

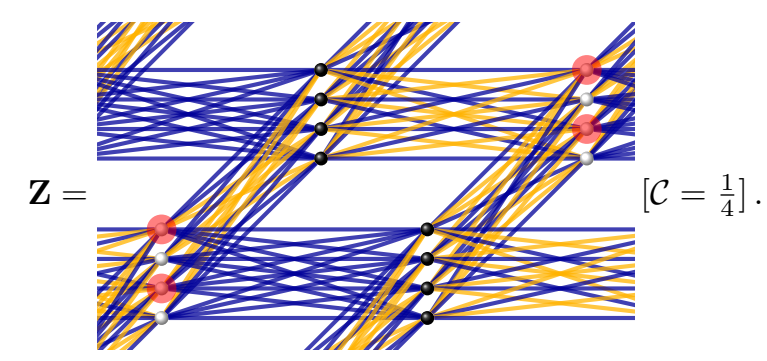

After doing so, connections between lattice "macronodes" (i.e., groups of four nodes) look like this:

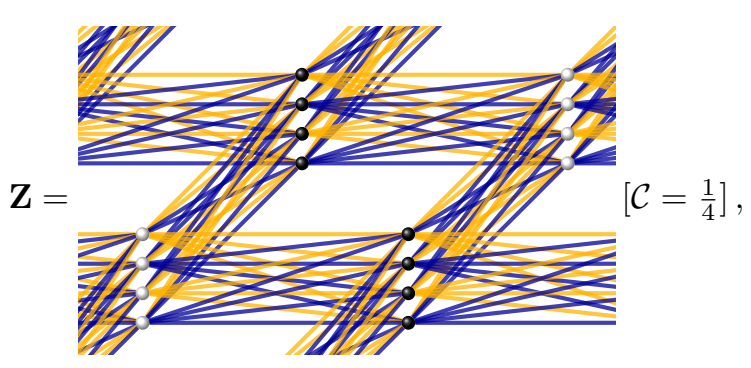

This graph is also bipartite (with white and black nodes, as shown) and satisfies $\mathbf{G}^{2}=\mathbf{G}^{\mathrm{T}} \mathbf{G}=\mathbf{I}$ everywhere except at a boundary.

The GPEPS construction for this state proceeds in two steps. We start with the GPEPS construction for a grid of unattached, crisscrossing quantum wires:

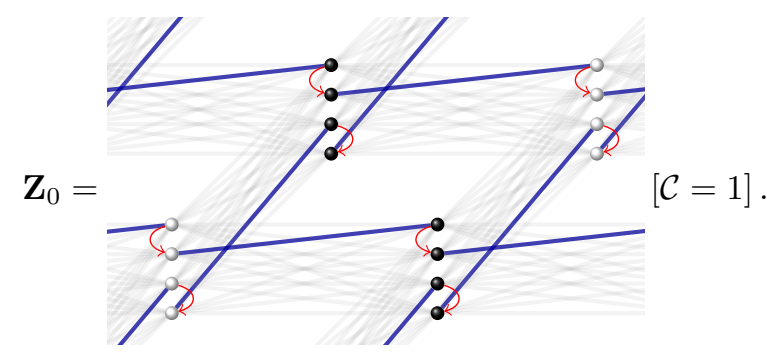

We then apply two additional beamsplitter interactions to $\mathbf{Z}_{0}$ :

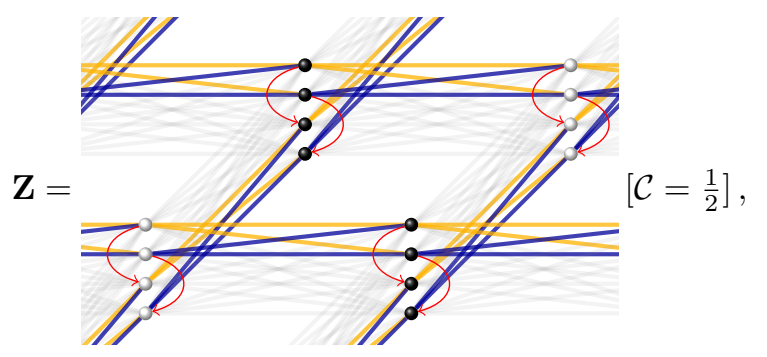

which results in the desired graph, shown in Eq. 25. This state can be projected down to an ordinary square lattice by measuring $\hat{q}$ on the top three nodes of each macronode (with an implicit phase shift by $-\frac{\pi}{2}$ on all white nodes; see Sec- 

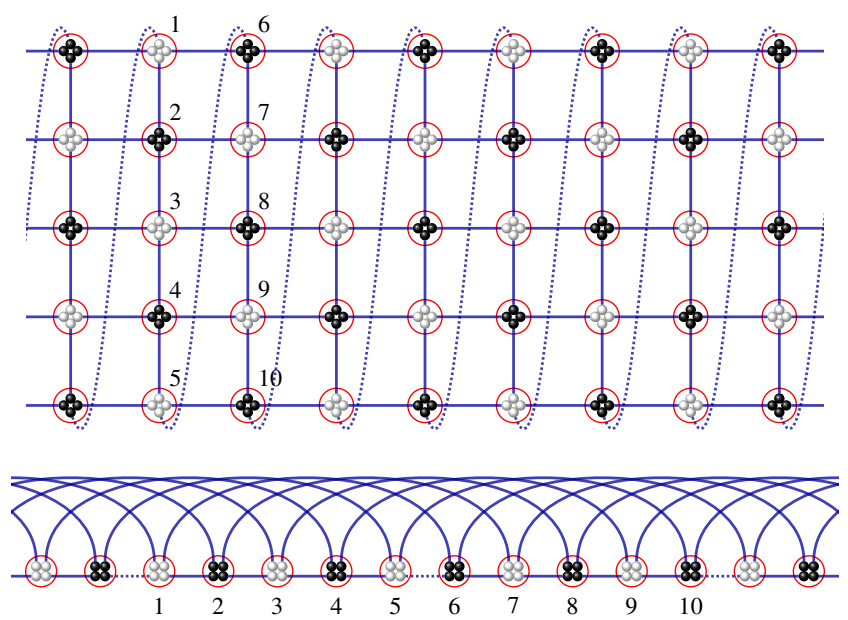

FIG. 3. Two equivalent representations of the GPEPS topology of the temporal-mode square-lattice CV cluster state illustrated in Eq. 25, adapted from Figure 3 in Ref. [10]. Two-mode squeezed states are arranged as in Eq. 26, while red circles represent the four beamsplitter transformations indicated in Eqs. 26] and 27) for each macronode, as well as the projection using $\hat{q}$ measurements on three of the micronodes within each, eventually resulting in the graph of Eq. 28. The two-dimensional square-lattice graph (top) is formally infinite in one dimension but finite in the other. This graph can be redrawn as a multiply threaded infinite line graph (bottom). There are $M$ additional threadings of the line graph that pass through macronodes $M$ units apart, resulting in a square lattice with vertical dimension $M$. Note that $M$ must be odd to ensure the graph is bipartite. (As shown, $M=5$.) The dotted links represent additional edges that would make the linear version translationally symmetric and equivalent to a square lattice on a cylinder with one unit of shear in the longitudinal direction. Such a family of graphs would still be universal for one-way QC because we can measure $\hat{q}$ on every $M$ th node to delete it (and its links) and "unfold" the graph into an ordinary square lattice with a vertical dimension of $(M-1)$ [6].

tion IIC):

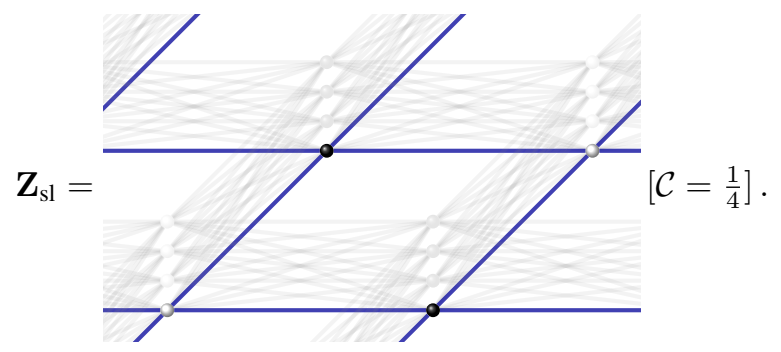

Just like for the quantum wire, this projection is useful for proving universality of the resulting state, but it may not strictly be necessary. Instead, it may be possible to manipulate quantum information encoded within a macronode as a whole (but still using only local measurements) and/or to use the additional connections for error correction. Work in this direction is ongoing.

We can use the techniques from the single-QND-gate method [10] to design a temporal-mode encoding of this state. The way this was done in that scheme was to "roll up" the

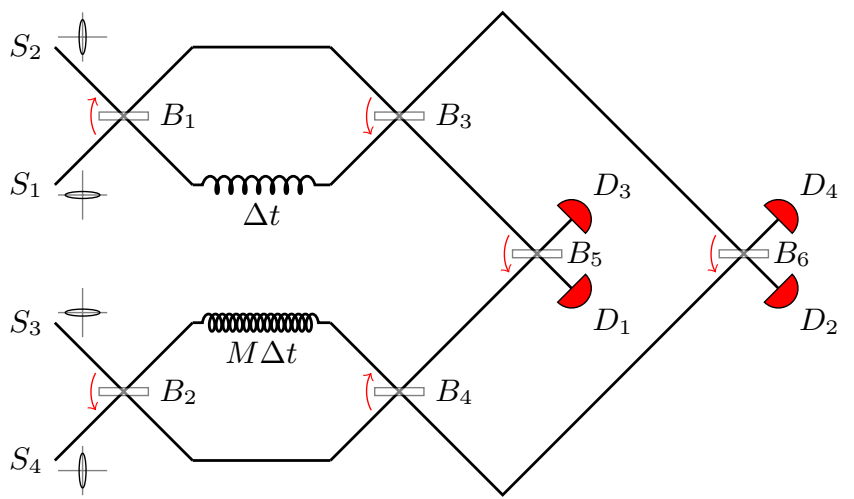

FIG. 4. Temporal-mode GPEPS construction of a square-lattice CV cluster state using passive squeezing and linear optics. Two copies of the quantum-wire setup from Figure 2 are used to generate the lattice. The upper one has the ordinary delay of $\Delta t$ and corresponds to the vertical links in Figure 3 (top). The longer delay of $M \Delta t$ in the lower one gives the second threading of the wire and corresponds to the horizontal links in Figure 3 (top). Beamsplitters $B_{3}$ and $B_{4}$ implement the transformations indicated by red arrows in Eq. 26. (Red arrows point from the first node to the second in Eq. 18 for each beamsplitter.) Following this, the 50:50 beamsplitters $B_{5}$ and $B_{6}$ implement the transformations indicated by red arrows in Eq. 27, eventually resulting in the state with graph $\mathbf{Z}$ from Eq. 25]. The outputs head to four detectors, which implement the $\hat{q}$-measurements (phase shifted as appropriate for the white nodes; see Section II C) to project the state down to an ordinary square lattice, Eq. (28), as well as the adaptive measurement-based quantum algorithm to be implemented. The adaptiveness means that subsequent measurement bases generally must be chosen based on previous measurement outcomes. Most measurements will involve homodyne detection in a basis that must be calculated and updated before the arrival of the next pulse, but the ability to divert the beam to an efficient photon counter is also required for universal QC [6].

square lattice onto a cylinder with a one-unit shift in the longitudinal direction. This allows the cluster state to be generated by double threading a quantum wire. The original threading provides the vertical links, while additional connections at a spacing of $M$ nodes apart create the second dimension of the lattice - see Figure 3 This corresponds to a simple linear optics circuit, illustrated in Figure 4. As shown in Section II B. and as in the case of the quantum wire in Section III] the "contaminated" nodes at the very beginning of the lattice can be disconnected from the rest of the cluster state by appropriate measurements of $\hat{q}$ on neighboring nodes, just like they are in the single-QND-gate method [10].

\section{DISCUSSION}

The methods present here for generating a temporal-mode $\mathrm{CV}$ cluster state using offline squeezing and linear optics combine the best aspects of several proposals. The temporal-mode encoding of the single-QND-gate method [10] allows finite hardware to be used repeatedly by pulsing the single-mode squeezer. This method achieves the same result, allowing op- 
tical elements to be reused by encoding field modes in pulses of finite duration. The advantage of this proposal is that the need for inline squeezing $\left(\mathrm{C}_{Z}\right.$ gate) is eliminated in favor of offline squeezing only, which is sufficient to produce twomode squeezed states when combined with linear optics. This was the advantage of the linear optics method [11]. Finally, an extensible design combining the two was arrived at by considering the states generated in the single-OPO method [8]. These states have special properties-specifically, that their graphs are bipartite and self-inverse - that allow 50:50 beamsplitters to link the two-mode squeezed pairs together. Delay loops and phase shifts are the only other ingredients needed to generate the states.

Computing with the states is possible by measuring in $\hat{q}$ the extra "layers" of connections-which exist solely due to the use of macronodes in place of individual nodes, and three of the detectors in Figure 4 can be assigned simply to this purpose. On the other hand, it may be possible to use all four detectors in concert to perform CV one-way QC on modes that are distributed over the macronode. The optimal measurement scheme for using these states is an open area of research.

The motivation for this is the need for error correction. It has been known since the beginning [4] that finite-energy CV cluster states are, in some sense, inherently faulty due to their finitely squeezed nature. Recent results [28] show this faultiness to be persistent in the sense that simple encodings of qubits or other low-dimensional systems in a finite number of CV modes cannot eliminate the errors caused by finite squeezing. As emphasized in that paper, this does not mean that $\mathrm{CV}$ cluster states (made by this method or any other) are unusable for CV one-way QC. It just means that there is no "magic pill" that will eliminate the faultiness introduced by finite squeezing. Instead, error correction and fault tolerance must be addressed from the very beginning if any $\mathrm{CV}$ one-way $\mathrm{QC}$ scheme is to be scalable. While a fault tolerant threshold [1] for CV one-way QC is not yet known to exist, promising results for qubit-based cluster states [35] and the known ability to use CVs for fault-tolerant QC in other contexts [36] inspire confidence that such a threshold will eventually be found.

All possible schemes for QC using CV cluster states will run into this problem because the faultiness of the states is due to finite energy constraints. The current scheme is no exception. Nevertheless, there are several reasons to believe this is a significant improvement over other optical schemes to date. The main reason is that the temporal-mode encoding limits the need for long-time coherence of the state since more cluster is prepared as previous pieces are consumed by the detectors. The main theoretical limitation for the current scheme is the long delay loop in Figure 4 , the length of which sets the width $M$ of the lattice. While computation can theoretically increase indefinitely in the horizontal dimension, the number of horizontal quantum wires linked together (and thus the number of encoded qubits or other systems) will be limited by how long this delay loop can be made without losing the ability to phaselock and modematch the interactions at the subsequent beamsplitters. Loss and the finite coherence length of the lasers also come into play, since at least $2 M$ pulses from each of the four squeezers must continue to exist coherently at all times. Despite these restrictions, the simplicity of the setup presented in Figure 4 makes it highly appealing for new experimental work. Experiments to date, which employ the original linear optics method [11], are currently limited to about four modes [19-22].

The main avenues for new research in this area include, first and foremost, the experimental implementation of this scheme. Beyond that, there remain important questions about how best to adapt to the use of macronodes in place of individual nodes in the cluster. This was alluded to several times in the text, and the issue relates both to efficiency of implementation and also to error correction, fault tolerance, and more efficient use of the squeezing resources available. Possible extensions include higher-dimensional graphs, such as the 3D graphs, which are useful in topological one-way QC using qubit-based cluster states and result in very high fault-tolerant thresholds [35]. The practical limitations of using only finite hardware mean that there is a maximum number of encoded qubits (i.e., a maximum $M$ ) beyond which the scheme will cease to be feasible, if for no other reason than the coherence length of the laser is exceeded. At this point, either the quantum wire setup from Figure 2 or the one for the square lattice from Figure 4 will need to be concatenated with another such system in order to scale up further. The same also applies to very long computations if we want them to be done fault tolerantly since longer computations will necessarily require larger encodings [28], which reduces to the problem above. The precise limitations and means of concatenation remain open areas of research. Despite this, given the current state of the art in linear optics, we have good reason to believe that the method proposed here will provide an important new avenue for experimental implementation of $\mathrm{CV}$ one-way QC.

\section{ACKNOWLEDGMENTS}

I thank Tim Ralph, Steve Flammia, Peter van Loock, Olivier Pfister, and Akira Furusawa for helpful discussions and suggestions. I am also grateful to Tim Ralph, Gerard Milburn, The University of Queensland, and the ARC Centre of Excellence for Quantum Computer Technology for support during visits that contributed to this work. Research at Perimeter Institute is supported by the Government of Canada through Industry Canada and by the Province of Ontario through the Ministry of Research \& Innovation.
[1] M. A. Nielsen and I. L. Chuang, Quantum Computation and Quantum Information (Cambridge, 2000).
[2] R. Raussendorf and H. J. Briegel, "A one-way quantum computer,” Phys. Rev. Lett. 86, 5188 (2001). 
[3] H. J. Briegel and R. Raussendorf, "Persistent Entanglement in Arrays of Interacting Particles," Phys. Rev. Lett. 86, 910 (2001).

[4] N. C. Menicucci et al., "Universal Quantum Computation with Continuous-Variable Cluster States," Phys. Rev. Lett. 97, 110501 (2006).

[5] J. Zhang and S. L. Braunstein, "Continuous-variable Gaussian analog of cluster states," Phys. Rev. A 73, 032318 (2006).

[6] M. Gu et al., "Quantum Computing with Continuous-Variable Clusters,” Phys. Rev. A 79, 062318 (2009).

[7] M. A. Nielsen, "Cluster-State Quantum Computation," Rep. Math. Phys. 57, 147 (2006).

[8] N. C. Menicucci, S. T. Flammia, and O. Pfister, "One-Way Quantum Computing in the Optical Frequency Comb," Phys. Rev. Lett. 101, 130501 (2008).

[9] S. T. Flammia, N. C. Menicucci, and O. Pfister, "The Optical Frequency Comb as a One-Way Quantum Computer,' J. Phys. B 42, 114009 (2009).

[10] N. C. Menicucci, X. Ma, and T. C. Ralph, "Arbitrarily Large Continuous-Variable Cluster States from a Single Quantum Nondemolition Gate,” Phys. Rev. Lett. 104, 250503 (2010).

[11] P. van Loock, C. Weedbrook, and M. Gu, "Building Gaussian cluster states by linear optics," Phys. Rev. A 76, 032321 (2007).

[12] M. A. Nielsen, "Optical Quantum Computation Using Cluster States," Phys. Rev. Lett. 93, 040503 (2004).

[13] D. E. Browne and T. Rudolph, "Resource-Efficient Linear Optical Quantum Computation,” Phys. Rev. Lett. 95, 010501 (2005).

[14] L.-M. Duan and R. Raussendorf, "Efficient Quantum Computation with Probabilistic Quantum Gates,” Phys. Rev. Lett. 95, 080503 (2005).

[15] H.-A. Bachor and T. C. Ralph, A Guide to Experiments in Quantum Optics (Wiley, Weinheim, 2004).

[16] S. L. Braunstein, "Squeezing as an irreducible resource," Phys. Rev. A 71, 055801 (2005).

[17] B. Yurke, "Optical back-action-evading amplifiers," J. Opt. Soc. Am. B 2, 732 (1985).

[18] J. Yoshikawa et al., "Demonstration of a quantum nondemolition sum gate," Phys. Rev. Lett. 101, 250501 (2008).

[19] X. Su et al., "Experimental Preparation of Quadripartite Cluster and Greenberger-Horne-Zeilinger Entangled States for Continuous Variables,” Phys. Rev. Lett. 98, 070502 (2007).

[20] H. Yonezawa and A. Furusawa, "Continuous-variable quantum information processing with squeezed states of light," Optics and Spectroscopy 108, 288 (2010).

[21] M. Yukawa et al., "Experimental generation of four-mode continuous-variable cluster states," Phys. Rev. A 78, 012301 (2008).
[22] R. Ukai et al., "Demonstration of unconditional one-way quantum computations for continuous variables," arXiv:1001.4860 [quant-ph] (2010).

[23] Y. Miwa et al., "Demonstration of Cluster State Shaping and Quantum Erasure for Continuous Variables," arXiv:1006.2225 [quant-ph] (2010).

[24] R. C. Pooser and O. Pfister, "Observation of triply coincident nonlinearities in periodically poled $\mathrm{KTiOPO}_{4}$," Opt. Lett. 30, 2635 (2005).

[25] M. Pysher et al., "Quasi-phase-matched concurrent nonlinearities in periodically poled KTiOPO4 for quantum computing over the optical frequency comb," Opt. Lett. 35, 565 (2010).

[26] M. Pysher et al., "Broadband amplitude squeezing in a periodically poled $\mathrm{KTiOPO}_{4}$ waveguide," Opt. Lett. 34, 256 (2009).

[27] S. L. W. Midgley et al., "Quadripartite continuous-variable entanglement via quadruply concurrent downconversion," arXiv:1002.2019 [quant-ph] (2010).

[28] M. Ohliger, K. Kieling, and J. Eisert, "Limitations of quantum computing with Gaussian cluster states," arXiv:1004.0081 [quant-ph] (2010).

[29] N. C. Menicucci, S. T. Flammia, and P. van Loock, "Graphical calculus for Gaussian pure states with applications to continuous-variable cluster states," arXiv:1007.0725 [quant-ph] (2010).

[30] J. Zhang, "Graphical description of local Gaussian operations for continuous-variable weighted graph states," Phys. Rev. A 78, 052307 (2008).

[31] J. Zhang, "Graphical rule of transforming continuous-variable graph states by local homodyne detection," arXiv:1006.3974 [quant-ph] (2010).

[32] R. Simon, E. C. G. Sudarshan, and N. Mukunda, "Gaussian pure states in quantum mechanics and the symplectic group," Phys. Rev. A 37, 3028 (1988).

[33] N. C. Menicucci et al., "Ultracompact generation of continuous-variable cluster states," Phys. Rev. A 76, 010302(R) (2007).

[34] H. Zaidi et al., "Entangling the optical frequency comb: simultaneous generation of multiple $2 \times 2$ and $2 \times 3$ continuous-variable cluster states in a single optical parametric oscillator," Laser Phys. 18, 659 (2008).

[35] R. Raussendorf, J. Harrington, and K. Goyal, "A fault-tolerant one-way quantum computer," Ann. Phys. (NY) 321, 2242 (2006).

[36] A. P. Lund, T. C. Ralph, and H. L. Haselgrove, "Fault-Tolerant Linear Optical Quantum Computing with Small-Amplitude Coherent States," Phys. Rev. Lett. 100, 030503 (2008). 